\title{
Direct Observation of Thermal Relaxation in Artificial Spin Ice
}

\author{
A. Farhan, ${ }^{1,2}$ P. M. Derlet, ${ }^{3}$ A. Kleibert, ${ }^{4}$ A. Balan, ${ }^{4}$ R. V. Chopdekar, ${ }^{1,4}$ M. Wyss, ${ }^{1,5}$ \\ J. Perron, ${ }^{1,2,6}$ A. Scholl, ${ }^{7}$ F. Nolting, ${ }^{4}$ and L. J. Heyderman ${ }^{1,2, *}$ \\ ${ }^{1}$ Laboratory for Micro- and Nanotechnology, Paul Scherrer Institute, 5232 Villigen PSI, Switzerland \\ ${ }^{2}$ Laboratory for Mesoscopic Systems, Department of Materials, ETH Zurich, 8093 Zurich, Switzerland \\ ${ }^{3}$ Condensed Matter Theory Group, NUM, Paul Scherrer Institute, 5232 Villigen PSI, Switzerland \\ ${ }^{4}$ Swiss Light Source, Paul Scherrer Institute, 5232 Villigen PSI, Switzerland \\ ${ }^{5}$ Swiss Nanoscience Institute, University of Basel, Klingelbergstrasse 82, 4056 Basel, Switzerland \\ ${ }^{6}$ Laboratoire de Chimie-Physique-Matière et Rayonnement (UMR 7614 UPMC/CNRS), \\ Université Pierre et Marie Curie, 75231 Paris Cedex 05, France \\ ${ }^{7}$ Lawrence Berkeley National Laboratory (LBNL), 1 Cyclotron Road, Berkeley, California 94720, USA
}

(Received 22 May 2013; revised manuscript received 2 July 2013; published 2 August 2013)

\begin{abstract}
We study the thermal relaxation of artificial spin ice with photoemission electron microscopy, and are able to directly observe how such a system finds its way from an energetically excited state to the ground state. On plotting vertex-type populations as a function of time, we can characterize the relaxation, which occurs in two stages, namely a string and a domain regime. Kinetic Monte Carlo simulations agree well with the temporal evolution of the magnetic state when including disorder, and the experimental results can be explained by considering the effective interaction energy associated with the separation of pairs of vertex excitations.
\end{abstract}

DOI: 10.1103/PhysRevLett.111.057204

PACS numbers: 75.10.Hk, 75.70.- $-\mathrm{i}, 75.78 .-\mathrm{n}$

Relaxation phenomena in various condensed matter systems, including dielectrics [1], glassy systems [2], and proteins [3], have long caught the attention of the research community. While studies of such relaxation processes involved measurements of macroscopic quantities, the recent creation of artificial spin systems has allowed the dynamics to be inspected microscopically [4,5]. A prominent example of such systems is artificial spin ice, which consists of dipolar-coupled nanoscale ferromagnets arranged in twodimensional frustrated geometries [6] and is considered to be a two-dimensional analogue to the naturally occurring pyrochlore spin ice [7]. Each nanomagnet is monodomain and elongated so that the magnetic moments point in one of two directions parallel to the island long axis, thus mimicking a single Ising spin. The main advantage of artificial spin ice systems is that their geometry can be tailored and their magnetic configurations can be directly visualized and investigated using appropriate imaging techniques [6,8-12]. However, due to the high blocking temperatures of the patterned nanomagnets, it has not been possible to observe thermal fluctuations in most of these systems and low-energy states could only partially be accessed using demagnetization protocols $[6,9,10]$. In terms of thermally active systems, in the past, investigations on superparamagnetic nanomagnets have been carried out [13-16], but temporally resolved real-space observations of thermally active artificial spin ice structures have been performed only recently [5].

In this Letter we now study thermally active extended arrays of artificial square ice [6] [see Fig. 1(a)], demonstrating at temperatures close to room temperature how such a system relaxes from a well-defined higher energy state to the lowest energy state, a behavior that compares well with our corresponding lattice kinetic Monte Carlo model. Specifically, we are able to observe the creation, separation, and annihilation of vertex excitations, which through their migration trigger the investigated relaxation processes. The results can be explained by an effective interaction energy (or string tension) that causes vertex excitations to separate and propagate, or converge and annihilate, depending on the background configuration through which they migrate.

For this work, a Permalloy (Ni83\%Fe17\%) wedge film on a silicon (100) substrate was patterned using electron beam lithography. Artificial square ice arrays consisting of nanomagnets, with length $L=470 \mathrm{~nm}$, width $W=$ $170 \mathrm{~nm}$, and center-to-center distance of nearest-neighbor nanomagnets $a=425 \mathrm{~nm}$, were fabricated, ranging in thickness from 0 to $20 \mathrm{~nm}$ over a distance of $3 \mathrm{~mm}$, with a $3 \mathrm{~nm}$-thick aluminum capping layer. Magnetic images [see Fig. 1(b)] were taken in a photoemission electron microscope (PEEM) [17], employing x-ray magnetic circular dichroism (XMCD) at the Fe $L_{3}$ edge [18]. The $\mathrm{XMCD}$ images were obtained with a total exposure time of six seconds (three seconds for each x-ray helicity). The dark and bright contrast in the obtained images is a measure of the orientation of the nanomagnet's magnetic moment relative to the $\mathrm{x}$-ray polarization vector. Nanomagnets with magnetic moments pointing toward the $\mathrm{x}$-ray polarization vector will appear dark, while nanomagnets with magnetic moments opposing the $\mathrm{x}$-ray polarization vector will appear bright [see Figs. 1(b) and 1(c)].

In order to investigate the relaxation processes, observations were performed at a film thickness of $3 \pm 0.3 \mathrm{~nm}$, 


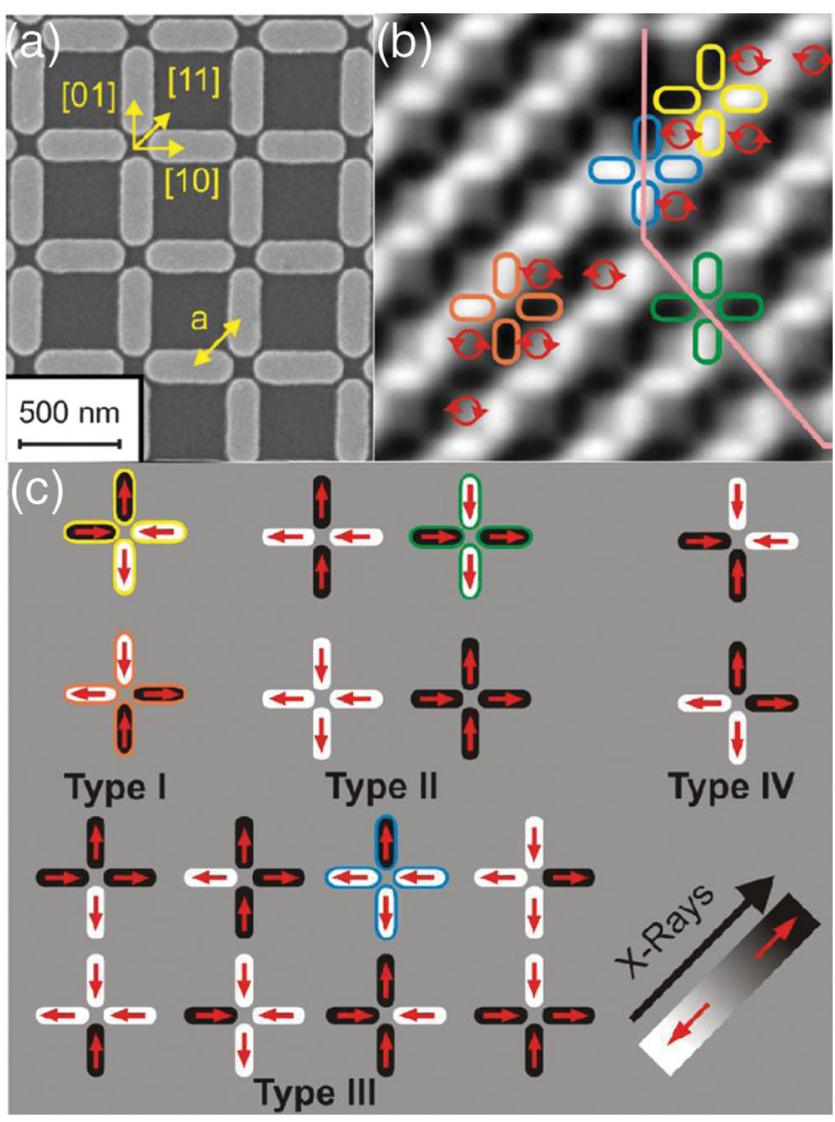

FIG. 1 (color). Artificial square ice. (a) Scanning electron microscope image $(a=425 \mathrm{~nm})$ and (b) associated XMCD image resolving vertex-type configurations detailed in (c). Highlighted in orange and yellow are type I vertices, and green and blue are a type II and a type III vertex, respectively. Present in (b) are two type I ground state domains of opposite chirality separated by a domain boundary (pink line) consisting of type II and type III vertices.

which allowed the details of evolution of the magnetic state to be recorded over a reasonable time scale. To understand the mechanisms behind the observed relaxation, it is important to first characterize the investigated system energetically. For artificial square ice, this is best done by considering the local vertex configurations [6], where each vertex is located at the center point of a cross consisting of four neighboring nanomagnets. The vertex type is given by the orientation of the moments of the nanomagnets and may be classified into four different types with increasing dipolar energy (type I to type IV) [see Fig. 1(c)]. The lowest energy configuration in an artificial square ice consists of an array of type I vertices, resulting in alternating clockwise and anticlockwise plaquettes. This ground state has a degeneracy of two, which can give rise to domains of opposite chirality [8]. While both type I and type II vertices have two moments pointing toward the vertex and two pointing away, obeying the so-called ice rule analogue to that found in the bulk systems [7], type II vertices have a higher energy since the interactions between the nanomagnets at a vertex in these two-dimensional systems are not equivalent $[6,19]$. Under an appropriate magnetic field, a magnetic configuration containing only type II vertices becomes the lowest energy configuration. Type III vertices can be considered as mobile thermal excitations and, as we will show, mediate configurational changes through their migration. Type IV vertices have all moments pointing in or all moments pointing out, and represent the most energetic configuration, which is never observed in our experiments.

An array of type II vertices was created in the artificial square ice after application of a saturating magnetic field (35 mT) along the negative [11] direction, and image acquisition was begun immediately after the magnetic field was switched off. Initially all moments point toward one direction (see bright XMCD contrast in Fig. 2(d) and the Supplemental Material [20]), and then the investigated system was found to pass through two distinct regimes while undergoing relaxation from the excited type II magnetic configuration to the lowest energy type I magnetic configuration (see Fig. 2). During the first regime we see isolated chains of type I vertices emerge within a type II vertex background via the creation of type III vertex pairs and their separation [see Figs. 2(a) and 2(d)]. Since this involves neighboring reversed island moments appearing as black lines, we refer to this regime as the string regime [see Figs. 2(a) and 2(d)]. During the string propagation, the reversal of nearest neighbor moments (in orthogonal nanomagnets) creates a chain of type I vertices within the initially saturated type II background. However, the less frequent reversal of next nearest neighbor moments (parallel islands at a vertex) results in trapped type II vertices, which have a different configuration to those forming the initial saturated state [see Fig. 2(d)] and become part of the boundaries in the domain regime [see Figs. 2(b) and 2(e), and the Supplemental Material [20]]. With an increasing number of strings, the ends of the strings meet (type III vertices annihilate) in either the same or across adjacent rows, and eventually form areas of (lowest energy) type I vertices. As there are two possible orientations for the type I vertex [8], such type I areas can be divided into two domain types of opposite chirality [see Fig. 1(b)]. This stage marks the beginning of the domain regime [see Fig. 2(b) and [20]], and subsequently these lowest energy type I domains of opposite chirality, separated by type II boundaries, evolve via type III vertex creation and propagation along the domain boundaries [see Fig. 2(e), and the Supplemental Material [20]]. Bigger domains expand at the cost of smaller domains through domain boundary movement until a uniform single domain [Fig. 2(c) and the Supplemental Material [20]] is achieved.

Taking a closer look at the vertex statistics with time [see Fig. 3(a)], we see that the string regime is characterized by a rapid increase (decrease) of type I (type II) vertices. In addition, the increasing number of strings in the early 


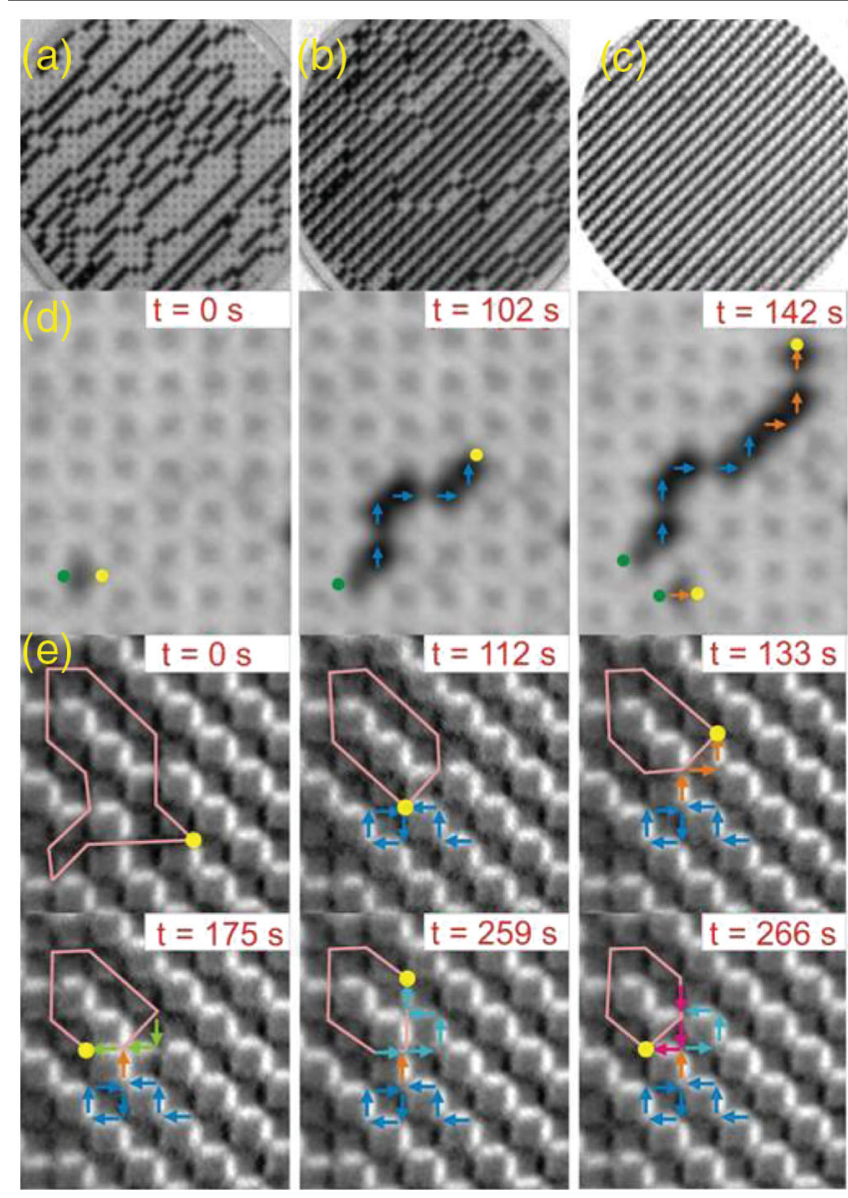

FIG. 2 (color). XMCD images of thermal relaxation in artificial spin ice: (a) string regime, (b) domain regime, and (c) the ground state (field of view $20 \mu \mathrm{m}$; see also the Supplemental Material [20]). Migration of type III vertices (green and yellow dots) in detail: (d) in the string regime, pairs of type III vertices are created and separate, and (e) in the domain regime, a type III vertex travels along various routes within a type II domain boundary (pink line) so that the bigger type I domain expands at the cost of the smaller domain. The path taken by the type III vertex is indicated with colored arrows and $a=425 \mathrm{~nm}$.

stages is reflected by an initial sharp increase in the number of type III vertices, which subsequently decrease in number as the string ends meet. In contrast, the domain regime is characterized by a general slow change in the vertex population, interspersed with small jumps in the number of type I (type II) vertices, associated with the creation and fast movement of type III vertices along a domain boundary. While this movement often occurs beyond the temporal limits of the XMCD imaging, several observations indicate that the movement of the type III vertices occurs via a sequential reversal of neighboring nanomagnets.

We now compare our experimental observations with kinetic Monte Carlo simulations. Here a simplified dipolar Hamiltonian is used, defining each nanomagnet to have either one of the two possible magnetic states with the moments aligned along the long axis of the nanomagnets.

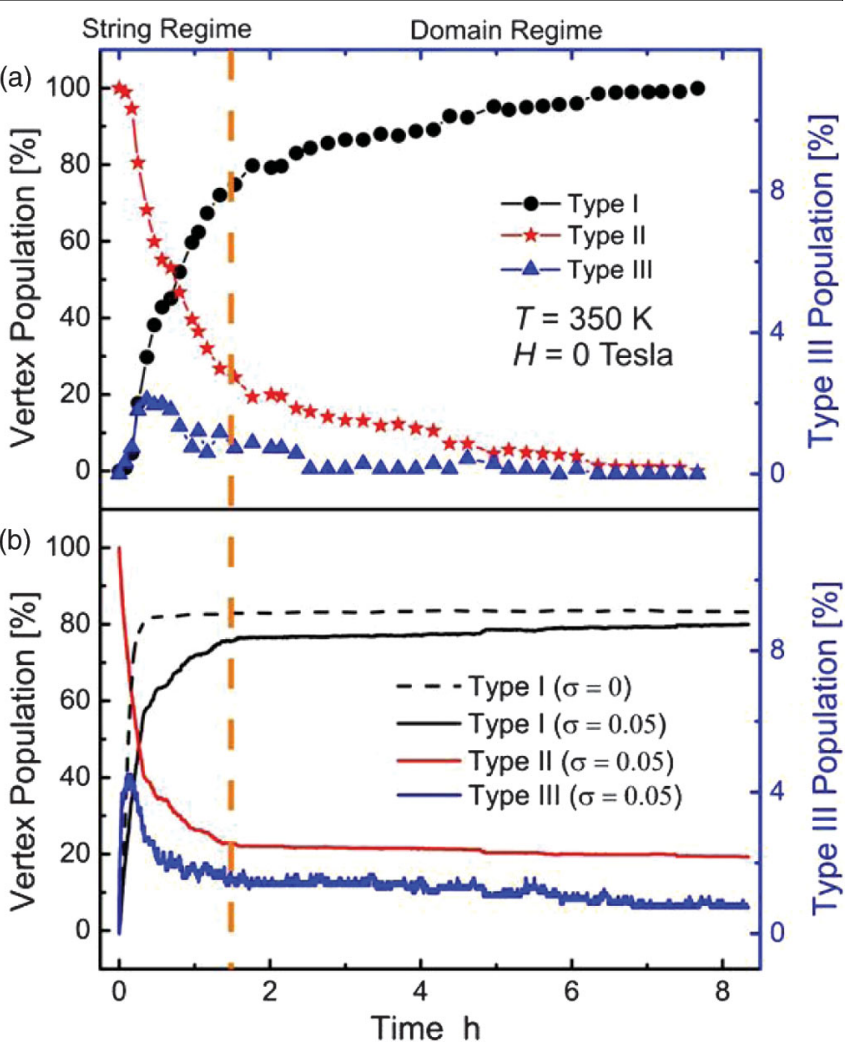

FIG. 3 (color). Temporal evolution of vertex-type population. (a) Experimental data obtained at a constant temperature of $350 \mathrm{~K}$, showing a $100 \%$ ground state ordering within eight hours. (b) Kinetic Monte Carlo simulation data for a Gaussian disorder in the intrinsic nanomagnet energy barrier with a standard deviation $\sigma=0.05$ and also for the case of no disorder, $\sigma=0$.

These mesoscopic magnetic moments interact via the magnetic dipolar interaction,

$V\left(\mathbf{r}_{i j}, \mathbf{m}_{i}, \mathbf{m}_{j}\right)=-\frac{\mu_{0}}{4 \pi r_{i j}^{3}}\left[3\left(\mathbf{m}_{i} \cdot \hat{\mathbf{r}}_{i j}\right)\left(\mathbf{m}_{j} \cdot \hat{\mathbf{r}}_{i j}\right)-\mathbf{m}_{i} \cdot \mathbf{m}_{j}\right]$,

where $\mathbf{r}_{i j}$ is the distance vector separating the $i$ th and $j$ th nanomagnets with magnetic moments $\mathbf{m}_{i}$ and $\mathbf{m}_{j}$, giving the final Hamiltonian $\sum_{i<j} V\left(\mathbf{r}_{i j}, \mathbf{m}_{i}, \mathbf{m}_{j}\right)$. Our calculations are performed for up to six nearest neighbors.

In order to realize the temporal evolution of the magnetization dynamics, the magnetic moment of each nanomagnet is assumed to have a reorientation rate $\nu$ given by the Arrhenius form $\nu_{0} \exp \left(-E / k_{B} T\right)$. Thus the magnetic moments are reoriented via thermal activation where $\nu_{0}$ is an intrinsic reorientation prefactor, and $E$ is a reorientation barrier energy equal to the sum of the intrinsic energy barrier of a nanomagnet, $E_{0}$, and half the dipolar energy gain associated with the particular moment reorientation [5]. For the calculation of the dipolar energy, we treat each nanomagnet as a point source, with each moment equal to the product of the nanomagnet's magnetization, 
$M$, and the experimental nanomagnet volume. The standard kinetic Monte Carlo technique [21] is then used to stochastically evolve the configuration and its physical time.

For reasonable agreement to experiment, values of $E_{0}=$ $1.05 \mathrm{eV}, M=350 \mathrm{kA} / \mathrm{m}$ and $\nu_{0}=0.5 \times 10^{12} \mathrm{~s}^{-1}$ were used in the kinetic Monte Carlo simulations. These are realistic values as explained in Ref. [5]. It was also found that a certain degree of disorder (variation in the nanomagnet anisotropy energy) was required in the kinetic Monte Carlo model to better reproduce the experimental observations of the increase in type I vertex population at the start of the string regime (see Fig. 3). Using a similar approach to that taken by Budrikis et al. [22], this was achieved by randomly varying the intrinsic energy barrier of each nanomagnet using a Gaussian distribution centered on $E_{0}$ with a standard deviation of $\sigma=0.05 \mathrm{eV}$. This is a lower value for $\sigma$ than that found in the literature for artificial spin ice [12] and other magnetic structures [23], which is likely to be a result of a more homogeneous microstructure at lower film thickness [24]. Disorder allows the system to explore many more pathways to the lowest energy state $[22,25]$, so requiring more time to develop from the perfect type II configuration into a multidomain low-energy configuration. Indeed, when we neglect disorder $(\sigma=0)$, we find an instantaneous initial increase in the type I vertex population, with the string regime being completed in only a few minutes (see Fig. 3(b), dashed black curve) rather than taking several hours to develop as seen in experiment. In addition, the inclusion of disorder causes the propagating type III vertices to periodically shift to neighboring rows, better matching the experimental observations [see Fig. 2(d)].

In general, the kinetic Monte Carlo simulations compare well with the experiment over the full relaxation process including both the string and domain regimes. This agreement was achieved by including dipolar interactions between first and second nearest neighbors only. The effect of adding the contributions from further nanomagnets up to six nearest neighbors $(6 a)$ was also investigated. However, this did not modify the temporal evolution in any significant way. In particular, there is good quantitative agreement in the temporal evolution of the vertex populations [Figs. 3(a) and 3(b)], where the initial 100\% type II vertex population rapidly decays as time evolves with a corresponding increase of the type I vertices. Inspection of the simulated domain regime occurring at later times reveals similar activity as that seen by Budrikis et al. [22], where the motion of domain boundaries (of type II vertices) is mediated by type III vertex creation, propagation, and annihilation.

For both experiment and simulation, the early stages of the string regime are characterized by the creation of isolated pairs of neighboring type III vertices [see Fig. 2(d)]. This is followed by further moment reorientations resulting in a rapid separation of the type III vertex

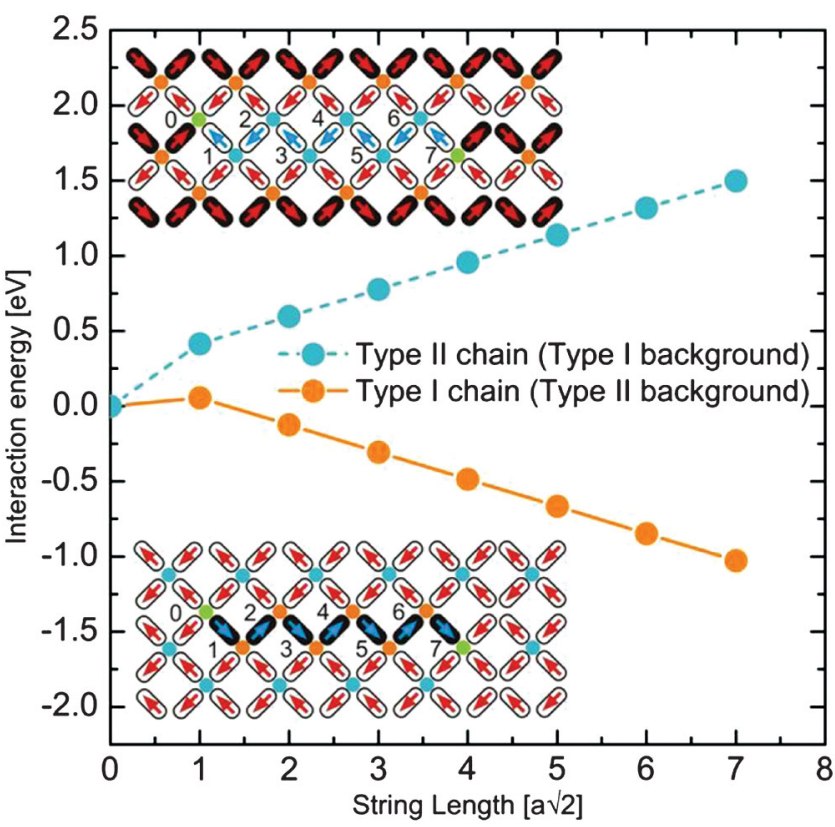

FIG. 4 (color). Interaction energy as a function of string length connecting two isolated type III vertices. Type I and II vertices are indicated by orange and blue dots, respectively, and the numbers in the schematics indicate the string length as the right-hand type III vertex follows a zigzag path.

pairs associated with the expansion of a chain of type I vertices between them along the [11] direction. This can be explained by considering the energy change as a function of the string length connecting the type III vertex pair in an otherwise perfect type II background, which may be readily calculated by determining the dipolar interaction energy between all nanomagnets, Eq. (1). The energy as a function of string length is plotted in Fig. 4, revealing that while the system initially increases its energy for the creation of the type III vertex pair, subsequent separation reduces the energy with a roughly linear decrease in energy as a function of their separation (see Fig. 4, orange line and bottom schematic). Thus, upon their creation, the type III vertex pair separate and rapidly move toward the edges of the array. This is in contrast to excitations above a type I background, where the separating type III vertex pair is now connected by a chain of type II vertices. Again there is a nearly linear change in energy, but now with a positive gradient (see Fig. 4, dashed blue line and upper schematic). Indeed, in the experiment we see that as soon the system falls into a single ground state domain consisting only of type I vertices, no further configurational changes are observed. This is an example of the confining potential associated with the type III vertices occurring in two dimensional artificial square ice systems that dominates over the negligible Coulomb interaction between charged vertex pairs $[26,27]$.

In conclusion, the realization of thermally active artificial spin ice at room temperature means that we now have access to model systems that are closer in nature to their 
three dimensional counterparts [7], and allow the magnetic configurations during relaxation to be detected in microscopic detail. This next generation of thermally active artificial spin ice systems offers the exciting prospect of direct observations of various relaxation phenomena. Under controllable thermal equilibrium conditions, this will also allow the long sought-after observation of phase transitions [28-30]. In terms of applications, the control of vertex excitations [11,12,31] will bring novel spintronic devices that make specific use of their thermally active magnetic properties.

The authors would like to thank Juri Honegger for technical support. This work was supported by the Swiss National Science Foundation and the Swiss Nanoscience Institute, Basel, Switzerland. Part of this work was performed at the Swiss Light Source, Paul Scherrer Institute, Villigen, Switzerland and the Advanced Light Source, Lawrence Berkeley National Laboratory (LBNL), Berkeley, California.

*laura.heyderman@psi.ch

[1] M. F. Shlesinger and E. W. Montroll, Proc. Natl. Acad. Sci. U.S.A. 81, 1280 (1984).

[2] R. G. Palmer, D. L. Stein, E. Abrahams, and P.W. Anderson, Phys. Rev. Lett. 53, 958 (1984).

[3] M. Levantino, A. Cupane, L. Zimányi, and P. Ormos, Proc. Natl. Acad. Sci. U.S.A. 101, 14402 (2004).

[4] Y. Han, Y. Shokef, A. M. Alsayed, P. Yunker, T.C. Lubensky, and A. G. Yodh, Nature (London) 456, 898 (2008).

[5] A. Farhan, P. M. Derlet, A. Kleibert, A. Balan, R. V. Chopdekar, M. Wyss, L. Anghinolfi, F. Nolting, and L. J. Heyderman, Nat. Phys. 9, 375 (2013).

[6] R. F. Wang, C. Nisoli, R. S. Freitas, J. Li, W. McConville, B. J. Cooley, M. S. Lund, N. Samarth, C. Leighton, V. H. Crespi, and P. Schiffer, Nature (London) 439, 303 (2006).

[7] M. J. Harris, S. T. Bramwell, D. F. McMorrow, T. Zeiske, and K. W. Godfrey, Phys. Rev. Lett. 79, 2554 (1997).

[8] J. P. Morgan, A. Stein, S. Langridge, and C. H. Marrows, Nat. Phys. 7, 75 (2011).

[9] Y. Qi, T. Brintlinger, and J. Cumings, Phys. Rev. B 77, 094418 (2008).

[10] E. Mengotti, L. J. Heyderman, A. Fraile Rodríguez, A. Bisig, L. Le Guyader, F. Nolting, and H. B. Braun, Phys. Rev. B 78, 144402 (2008).

[11] S. Ladak, D.E. Read, G. K. Perkins, L. F. Cohen, and W. R. Branford, Nat. Phys. 6, 359 (2010).
[12] E. Mengotti, L. J. Heyderman, A. Fraile Rodríguez, F. Nolting, R. V. Hügli, and H. B. Braun, Nat. Phys. 7, 68 (2011).

[13] W. Wernsdorfer, E. Bonet Orozco, K. Hasselbach, A. Benoit, B. Barbara, N. Demoncy, A. Loiseau, H. Pascard, and D. Mailly, Phys. Rev. Lett. 78, 1791 (1997).

[14] R. P. Cowburn, A. O. Adeyeye, and M. E. Welland, New J. Phys. 1, 16 (1999).

[15] S. Bedanta and W. Kleemann, J. Phys. D 42, 013001 (2009).

[16] S. Krause, G. Herzog, T. Stapelfeldt, L. Berbil-Bautista, M. Bode, E. Y. Vedmedenko, and R. Wiesendanger, Phys. Rev. Lett. 103, 127202 (2009).

[17] L. Le Guyader, A. Kleibert, A. Fraile Rodríguez, S. El Moussaoui, A. Balan, M. Buzzi, J. Raabe, and F. Nolting, J. Electron Spectrosc. Relat. Phenom. 185, 371 (2012).

[18] J. Stöhr, Y. Wu, B. D. Hermsmeier, M. G. Samant, G. R. Harp, S. Koranda, D. Dunham, and B. P. Tonner, Science 259, 658 (1993).

[19] G. Möller and R. Moessner, Phys. Rev. Lett. 96, 237202 (2006).

[20] See Supplemental Material at http://link.aps.org/ supplemental/10.1103/PhysRevLett.111.057204 for XMCD images demonstrating thermal relaxation in artificial spin ice.

[21] W. M. Young and E. W. Elcock, Proc. Phys. Soc. London 89, 735 (1966).

[22] Z. Budrikis, K. L. Livesey, J. P. Morgan, J. Akerman, A. Stein, S. Langridge, C. H. Marrows, and R.L. Stamps, New J. Phys. 14, 035014 (2012).

[23] F. Luo, L. J. Heyderman, H. Solak, T. Thomson, and M. E. Best, Appl. Phys. Lett. 92, 102505 (2008).

[24] J. W. Lau, R. D. Michael, S. H. Chung, J. O. Rantschler, V. Parekh, and D. Litvinov, Appl. Phys. Lett. 92, 012506 (2008).

[25] Z. Budrikis, P. Politi, and R. L. Stamps, Phys. Rev. Lett. 107, 217204 (2011).

[26] L. A. Mól, R. L. Silva, R. C. Silva, A. R. Pereira, W. A. Moura-Melo, and B. V. Costa, J. Appl. Phys. 106, 063913 (2009).

[27] R. C. Silva, F. S. Nascimento, L. A. S. Mól, W. A. MouraMelo, and A. R. Pereira, New J. Phys. 14, 015008 (2012).

[28] G. Möller and R. Moessner, Phys. Rev. B 80, 140409(R) (2009).

[29] G. W. Chern, P. Mellado, and O. Tchernyshyov, Phys. Rev. Lett. 106, 207202 (2011).

[30] D. Levis, L. F. Cugliandolo, L. Foini, and M. Tarzia, Phys. Rev. Lett. 110, 207206 (2013).

[31] R. V. Hügli, G. Duff, B. O’Conchuir, E. Mengotti, A. Fraile Rodríguez, F. Nolting, L. J. Heyderman, and H. B. Braun, Phil. Trans. R. Soc. A 370, 5767 (2012). 